\title{
IRRATIONAL CONNECTED SUMS AND THE TOPOLOGY OF ALGEBRAIC SURFACES
}

\author{
BY \\ RICHARD MANDELBAUM
}

\begin{abstract}
Suppose $W$ is an irreducible nonsingular projective algebraic 3-fold and $V$ a nonsingular hypersurface section of $W$. Denote by $V_{m}$ a nonsingular element of $|m V|$. Let $V_{1}, V_{m}, V_{m+1}$ be generic elements of $|V|$, $|m V|,|(m+1) V|$ respectively such that they have normal crossing in $W$. Let $S_{1 m}=V_{1} \cap V_{m}$ and $C=V_{1} \cap V_{m} \cap V_{m+1}$. Then $S_{1 m}$ is a nonsingular curve of genus $g_{m}$ and $C$ is a collection of $N=m(m+1) V_{1}^{3}$ points on $S_{1 m}$. By [MM2] we find that (*) $V_{m+1}$ is diffeomorphic to $\overline{V_{m}-T\left(S_{1 m}\right)} \cup_{\eta} \overline{V_{1}^{\prime}-T\left(S_{1 m}^{\prime}\right)}$ where $T\left(S_{1 m}\right)$ is a tubular neighborhood of $S_{1 m}$ in $V_{m}, V_{1}^{\prime}$ is $V_{1}$ blown up along $C, S_{1 m}^{\prime}$ is the strict image of $S_{1 m}$ in $V_{1}^{\prime}, T\left(S_{1 m}^{\prime}\right)$ is a tubular neighborhood of $S_{1 m}^{\prime}$ in $V_{1}^{\prime}$ and $\eta: \partial T\left(S_{1 m}\right)$ $\rightarrow \partial T\left(S_{m}^{\prime}\right)$ is a bundle diffeomorphism.

Now $V_{1}^{\prime}$ is well known to be diffeomorphic to $V_{1} \# N\left(-C P^{2}\right)$ (the connected sum of $V_{1}$ and $N$ copies of $C P^{2}$ with opposite orientation from the usual). Thus in order to be able to inductively reduce questions about the structure of $V_{m}$ to ones about $V_{1}$ we must simplify the "irrational sum" (*) above.

The general question we can ask is then the following:

Suppose $M_{1}$ and $M_{2}$ are compact smooth 4-manifolds and $K$ is a connected $q$-complex embedded in $M_{i}$. Let $T_{i}$ be a regular neighborhood of $K$ in $M_{i}$ and let $\eta: \partial T_{1} \rightarrow \partial T_{2}$ be a diffeomorphism:

Set $V=\overline{M_{1}-T_{1}} \cup \overline{M_{2}-T_{2}}$. How can the topology of $V$ be described more simply in terms of those of $M_{1}$ and $M_{2}$.

In this paper we show how surgery can be used to simplify the structure of $V$ in the case $q=1,2$ and indicate some applications to the topology of algebraic surfaces.
\end{abstract}

Introduction. Suppose $M_{1}, M_{2}$ are smooth compact 4-manifolds. Then the connected sum $M_{1} \# M_{2}$ is defined (see [KM, §2]) by removing 4-discs $D_{1}$ from $M_{1}$ and $D_{2}$ from $M_{2}$ and identifying $\partial \overline{M_{1}-D_{1}} \approx S^{3}$ with $\partial \overline{M_{2}-D_{2}}$ by means of an orientation-reversing diffeomorphism $h$. As noted in [KM] $M_{1} \# M_{2}=\overline{M_{1}-D_{1}} \cup \cup_{h} \overline{M_{2}-D_{2}}$ is in fact independent of the particular diffeomorphism used. Now a 4-disc $D_{1}$ in $M_{1}$ is in fact a regular neighborhood of a zero-cell " $p$ " in $M_{1}$. Suppose we "connect" two such manifolds along the boundary, not of a regular neighborhood of a point, but of a regular neighborhood of an embedded $n$-complex? In particular, suppose $K$ is

Received by the editors February 23, 1977 and, in revised form, November 10, 1977. AMS (MOS) subject classifications (1970). Primary 57D55, 57A15, 14 J99.

(C) 1979 American Mathematical Society 0002-9947/79/0000-0005/\$06.00 
a wedge of $k$ 1-spheres embedded in $M_{1}$ and $M_{2}$ and $V=\overline{M_{1}-N_{1}(K)} \cup_{\eta} \overline{M_{2}-N_{2}(K)}$, (where $N_{i}(K)$ is a regular neighborhood of $K$ in $M_{i}$ and $\eta: \partial N_{1} \rightarrow \partial N_{2}$ is some diffeomorphism). What can be said about the topology of $V$ ? In the general case this depends, of course, on the homotopy class of $K$ in $M_{i}$. If this is zero, that is, if $K$ is homotopic to zero in both $M_{1}$ and $M_{2}$ we can reduce $V$ to a connected sum.

In particular let $P=C P^{2}, Q=-C P^{2}$ (where - will denote reversed orientation) and let $k X=X \# \ldots \ldots X$ (k-times) for a manifold $X$. Then in the above situation, under some mild hypothesis on $\eta$ we have

Theorem A (See Proposition 2.2 and Theorem 2.3). Suppose $V=\overline{M_{1}-N_{1}} \cup \eta \overline{M_{2}-N_{2}}$ as above and $\eta$ is the restriction of an orientation reversing diffeomorphism $\phi: N_{1} \rightarrow N_{2}$ which induces the identity on $\pi_{1}(K)$. Then either

(a) $V \approx M_{1} \# M_{2} \# k\left(S^{2} \times S^{2}\right)$ or

(b) $V \approx M_{1} \# M_{2} \# k(P \# Q)$

(where " $\approx$ " is read "is diffeomorphic to").

Now suppose we consider 2-complexes. In particular, replace $K$ by an embedded 2-manifold $S$ in $M_{i}$ with tubular neighborhood $T_{i}$ and let

$$
X=\overline{M_{1}-T_{1}} \cup_{\phi} \overline{M_{2}-T_{2}}
$$

where $\phi$ is a bundle isomorphism of $\partial T_{1}$ and $\partial T_{2}$.

Now as opposed to the case of 1-complexes in 4-manifolds, even if the $M_{i}$ are simply-connected the nature of $X$ will, a priori, depend on the placement of $K$ in $M_{i}$.

Using either surgery or in some cases a combination of $\sigma$ and $\bar{\sigma}$-processes we can still obtain a good deal of information about $X$ even without assuming anything about the placement of $K$. In fact we have

TheOREM B (SeE Theorems 3.4 AND 3.5). If $X=\overline{M_{1}-T_{1}} \cup_{\phi} \overline{M_{2}-T_{2}}$ as above with the $M_{i}$ simply-connected and the $\phi$ orientation reversing, then:

(1) If a fiber $F$ of $\partial T_{1} \rightarrow S$ considered as a loop in $X$ is homotopic to zero, then either

$$
X \#\left(S^{2} \times S^{2}\right) \approx M_{1} \# M_{2} \# k\left(S^{2} \times S^{2}\right)
$$

or

$$
X \#(P \# Q) \approx M_{1} \# M_{2} \# k(P \# Q)
$$

where $k=$ minimal number of generators of $H_{1}(S, Z)$.

(2) If $M_{2}$ is obtained by blowing up a manifold $N$ by a $\sigma$-process at a point of a submanifold $\Sigma$ whose strict image in $M_{2}$ is $S$, then

$$
X \# P \approx M_{1} \# N \# k(P \# Q) \text {. }
$$


In our applications of Theorems $\mathrm{A}$ and $\mathrm{B}$ it is rare that we begin with manifolds $M_{1}, M_{2}$ and patch them together to get $X$ (or $V$ ) as above. The more usual situation is that we are given some manifold $X$ and by various means we demonstrate the existence of manifolds $M_{1}, M_{2}$ such that $X$ is obtained by patching the $M_{i}$ along some neighborhoods $T_{i}$. A very useful method for obtaining such 'irrational connected sum' representations is provided by the theorem of $\$ 2$ of [MM2]. In particular, we have

Theorem C (SeE TheORems 3.7 AND 3.8). Suppose $W$ is a compact complex 3-manifold and $V, X_{1}, X_{2}$ are compact complex submanifolds of $W$ with normal intersection such that as divisors on $W, V$ is linearly equivalent to $X_{1}+X_{2}$. Suppose $S=X_{1} \cap X_{2}$ is connected, $V \cdot X_{1} \cdot X_{2}=n>0$ and the $X_{i}$ are simply connected. Then

$$
\text { (1) } V \approx \overline{X_{1}-T_{1}} \cup \overline{X_{2}^{\prime}-T_{2}^{\prime}}
$$

where $X_{2}^{\prime}$ is $X_{2}$ blown up at the n-points of $V \cap X_{1} \cap X_{2}$ and $T_{1}, T_{2}^{\prime}$ are tubular neighborhoods of $S$, respectively $S^{\prime}\left(S^{\prime}=\right.$ the strict image of $\left.S\right)$, in $X_{1}$, respectively $X_{2}^{\prime}$.

(2) $V \# P \approx X_{1} \# X_{2} \#(n-1) Q \# 2 g(P \# Q)$

where $g=$ genus of $S$.

In $\$ 4$ we put the above results to work in investigating the structure of simply connected algebraic surfaces.

It may be recalled that if $M$ is a simply-connected compact 4-manifold then [see Mi] the homotopy type of $M$ is completely determined by the congruence class of the quadratic form $q_{M}$ given by the cup product (or dually by homology-intersection) on $H^{2}(M, Z)$ (or $H_{2}(M, Z)$ ). In [W2] Wall shows that $q_{M}$ in fact determines $M$ up to $h$-cobordism and, by a modification of Smale's results in higher-dimensions, that $M$ is determined by $q_{M}$ up to diffeomorphism modulo connected sums of $S^{2} \times S^{2}$ s.

That is, by [W2] if $M_{1}, M_{2}$ are simply-connected compact 4-manifolds with $q_{M_{1}}$ congruent to $q_{M_{2}}$, then for some integer $k, M_{1} \# k\left(S^{2} \times S^{2}\right) \approx$ $M_{2} \# k\left(S^{2} \times S^{2}\right)$.

If $M$ is a 4-manifold diffeomorphic to $a P \# b Q$ for some integers $a, b$ we say that $M$ is completely decomposable. As noted in [MM1, §0] a consequence of Wall's result is that for any simply-connected 4-manifold there exists an integer $k$ such that $M \#(k+1) P \# k Q$ is completely decomposable.

If $M \# P$ is completely decomposable then we shall say that $M$ is almost completely decomposable (abbreviated as $M$ is A.C.D.). Note that if $M$ is A.C.D. it is of course simply-connected.

The main result of [MM1] was that any nonsingular complex hypersurface 
of $C P^{3}$ was almost completely decomposable.

In $\$ 4$ we generalize this result to other classes of simply-connected algebraic surfaces. Among other results we have

Theorem D (See Theorem 4.2 and Corollaries 4.3, 4.5). Suppose $X$ is an almost completely decomposable algebraic surface and $C$ is an irreducible nonsingular hypersurface section of $X$. Let $W \stackrel{\pi}{\rightarrow} X$ be the projective bundle over $X$ obtained by compactifying the line bundle $E=[k C](k>0)$. Then:

(1) If $V$ is an irreducible nonsingular hypersurface section of $W$ then $V$ is A.C.D.

(2) If $V$ is a nonsingular irreducible subvariety of $W$ such that $\pi \mid V: V \rightarrow X$ is a pure $n$-fold branched cover of $X$ then $V$ is A.C.D.

(3) If $V$ is a nonsingular $k$-fold cyclic branched cover of $X$ with branch locus $M$ linearly equivalent to $k C$, for some $k>0$ then $V$ is A.C.D.

In particular, the nonsingular 'double planes' are all A.C.D.

In the remainder of this article we will adopt the following conventions and notations.

All of our manifolds will be smooth. Furthermore when we construct manifolds by a 'cutting and pasting' technique we shall assume the resulting 'manifolds with corners' are smoothed to manifolds or manifolds with boundary by the process noted in $[\mathbf{K M}]$ or $[\mathbf{M z}]$. Thus for example if $D^{q}$ is a closed $q$-disc then it will make sense in terms of our smoothing, to say $D^{n} \approx D^{p} \times D^{n-p}$.

When we speak of regular neighborhoods or complexes in a manifold we shall be tacitly assuming that the manifold is given with a triangulation making such terms meaningful. Similarly tubular neighborhoods will be understood as referring to some underlying Riemannian metric.

We use the definition of $\sigma$ and $\bar{\sigma}$-process on an arbitrary 4-manifold enunciated in [MM1, Introduction] and elaborated on in [MM2]. For the convenience of the reader we recall that definition here:

Suppose $M$ is a 4-manifold and $p \in M$. Let $U$ be a small neighborhood of $p$ and take local coordinates on $U$ making it into a domain in $\mathbf{C}^{2}$. Perform a classical $\sigma$-process (blowing up, quadratic transformation, see [Sh] for definition) on $p \in U \subset C^{2}$ and call the resulting manifold $M^{\prime}$. Then if $M$ is oriented and the complex orientation on $U$ coincides with the induced orientation $U$ inherits from $M$ then we say $M^{\prime}$ is obtained by a $\sigma$-process of $p \in M$. If the two orientations are opposite we shall say $M$ is obtained by a $\bar{\sigma}$ process from $M$. (If $M$ is not orientable then the two notions coincide.) We note that a $\sigma$-operation changes $M$ into $M \# Q$ by introducing a new 2-sphere $L$ with self-intersection -1 while a $\bar{\sigma}$-operation changes $M$ into 
$M \# P$ with the resulting 2-sphere thereby introduced having self-intersection +1 . We sometimes will speak of the projective closure of the complex vector bundle $E \rightarrow M$ over some manifold $M$. By this we refer to the projective bundle $\mathscr{P}(E \oplus 1)$.

The first result of $\$ 3$ of [W1] will be used repeatedly in our work and we single it out as

THEOREM W (SEE [W1, §§1-3]). Let $N$ be a compact four manifold and suppose $C$ is a loop in $N$ homotopic to zero. Let $f: S^{1} \times D^{3} \rightarrow N$ be an embedding with $f\left(S^{1} \times\{0\}\right)=C$ and set $T=\operatorname{Im}(f)$.

Suppose $N^{\prime}$ is the manifold obtained by surgering $N$ along $T$. Then

(1) either $N^{\prime} \approx N \#\left(S^{2} \times S^{2}\right)$ or $N^{\prime} \approx N \#(P \# Q)$ and if $N$ is of odd type, then

(2)

$$
N \#\left(S^{2} \times S^{2}\right) \approx N \#(P \# Q) \approx N^{\prime} .
$$

As the proof of the first part of the above result is short and introduces other notions used later we give a brief sketch of it here.

By [W1, Lemma 4] and [RS, Theorem 7.10] since $C$ is homotopic to zero we may assume that it spans an embedded 2-disc, and lies in the interior of a 4-disc $D$ of $N$. Thus $N^{\prime}=N-\{$ 4-disc $\} \cup D-T \cup D^{2} \times S^{2}$. But $D-T$ $\approx D^{2} \times S^{2}-\{4$-disc $\}$ so that $D-T \cup D^{2} \times S^{2}$ is diffeomorphic to either $S^{2} \times S^{2}-\{4$-disc $\}$ or to $S^{2} \times S^{2}-\{4$-disc $\}$ (where $S^{2} \times S^{2}$ is the unique nontrivial $S^{2}$-bundle over $\left.S^{2}\right)$. Thus $N^{\prime} \approx N \#\left(S^{2} \times S^{2}\right)$ or $N^{\prime} \approx$ $N \#\left(S^{2} \times S^{2}\right)$. But as in [W1, Lemma 1] and [S, pp. 135,136] $S^{2} \times S^{2} \approx$ $P \# Q$. This concludes part (1).

Part (2) is just Corollary 1 of $\S 3$ of [W1]. We recall that the statement ' $N$ is of odd type' is equivalent to the existence of some homology 2-class $D$ in $N$ with $D^{2}$ odd.

Our general references for notions from complex analysis and algebraic geometry are [GR], [Sh] and for PL-topology [RS], [H].

I would like to thank the Institute des Hautes Études Scientifiques for their gracious hospitality during the period when this work was conceived. I would also like to thank John Morgan, Robert Connelly and Laurent Siebenmann for many helpful conversations during my stay at I.H.É.S., and especially for introducing me to some of the tools and methods of PL-topology. I would especially like to thank Boris Moishezon for initiating me into the study of the topology of algebraic surfaces and for all his help in answering my incessant questions in this area.

Lastly by way of explanation of the title we can think of manifolds patched together via neighborhoods of things other than points as being 'irrational connected sums' of such manifolds. 
2. Connected sums along 1-complexes. We now turn to the first question raised in the introduction, namely what is the structure of the manifold obtained by joining two manifolds together via the boundary of regular neighborhoods of 1-complexes in each of them. We begin with a preliminary lemma.

LEMMA 2.1. Suppose $M$ is a 4-manifold and $K$ is the image of an embedding of $\bigvee_{\alpha=1}^{n} S_{\alpha}^{1}$ in $M$. Let $T$ be a closed regular neighborhood of $K$ and set $H=\partial T$.

Let $\left\{e_{\alpha}\right\}, \alpha=1, \ldots, n$, be a collection of disjointly embedded 1-spheres in $H$ with $e_{\alpha}$ homotopic to $\operatorname{Im}\left(S_{\alpha}^{1} \hookrightarrow M\right)$.

Let $\left\{B_{\alpha}\right\}, \alpha=1, \ldots, n$, be a collection of disjoint 2-handles $\approx D^{2} \times D^{2}$ and suppose for each $\alpha, \phi_{\alpha}:\left(S^{1} \times D^{2}\right)_{\alpha} \subset \partial B_{\alpha} \rightarrow H$ is a diffeomorphism into $H$ with $\phi_{\alpha}\left(S^{1} \times 0\right)=e_{\alpha}$ such that $T \cup_{\phi} B$ is a 4-ball, where $\phi=\cup \phi_{\alpha}$ and $B=\cup B_{\alpha}$.

Now suppose $\omega_{\alpha}:\left(S^{1} \times D^{3}\right)_{\alpha} \rightarrow M$ are diffeomorphisms, $\alpha=1, \ldots, n$, with $\omega_{\alpha} \mid\left(S^{1} \times D^{2}\right)_{\alpha}=\phi_{\alpha}$ [where $D^{2} \hookrightarrow \partial D^{3}$ is the upper hemisphere] and with disjoint images. Let $\chi(M)$ be the manifold obtained by doing surgery on $M$ along the $e_{\alpha}$ with framing equivalent to $\omega_{\alpha}$. Let $M^{\prime}=\overline{M-T} \cup_{\phi} B$. Then

$$
M^{\prime} \approx \overline{\chi(M)-\{4-\text { ball }\}} .
$$

Proof. Let $T_{\alpha}=\omega_{\alpha}\left(S^{1} \times D^{3}\right)$. By isotoping $T$ slightly we may suppose that $T_{\alpha} \subseteq T$ with $\overline{T-T_{\alpha}} \approx T$.

Now

$$
\begin{gathered}
\chi(M)=\overline{M-\left(\bigcup_{\alpha} T_{\alpha}\right)} \cup_{\omega} \bigcup_{\alpha}\left(D^{2} \times S^{2}\right)_{\alpha}, \\
\omega=\bigcup \omega_{\alpha} \mid \bigcup \partial\left(S^{1} \times D^{3}\right)_{\alpha}=\bigcup \partial\left(D^{2} \times S^{2}\right)_{\alpha} \rightarrow M .
\end{gathered}
$$

Identify $B_{\alpha}$ with $\left(D^{2} \times S_{+}^{2}\right)_{\alpha}$, where $S_{+}^{2}, S_{-}^{2}$ are the closed upper and lower hemisphere of $S^{2}$, respectively, and note that $\omega_{\alpha} \mid\left(S^{1} \times S_{+}^{2}\right)_{\alpha}=\phi_{\alpha}$. Let $\tilde{\phi}_{\alpha}=$ $\omega_{\alpha} \mid\left(S^{1} \times S_{-}^{2}\right)_{\alpha}, \tilde{\phi}=\cup \tilde{\phi}_{\alpha}, \tilde{B}_{\alpha}=\left(D^{2} \times S_{-}^{2}\right)_{\alpha}, \tilde{B}=\cup \tilde{B}_{\alpha}$. Let

$$
\chi(T)=\overline{T-\left(\bigcup_{\alpha} T_{\alpha}\right)} \cup_{\tilde{\phi}} \tilde{B} \text {. }
$$

It is clear that

$$
\chi(T) \approx T \cup_{\phi} B \approx\{4 \text {-ball }\}
$$

and therefore

$$
\overline{\chi(M)-\{\text { 4-ball }\}}=\overline{\chi(M)-\chi(T)}=\overline{M-T} \cup_{\phi} B=M^{\prime}
$$

as desired. We now obtain 
Proposition 2.2. Let $M_{1}, M_{2}$ be compact 4-manifolds, set $K=\bigvee_{\alpha=1}^{n} S_{\alpha}^{1}$ and let $K_{i} \subset M_{i}$ be the image of an embedding of $K$ in $M_{i}, i=1,2$. Let $T_{i}$ be a regular neighborhood of $K_{i}$, set $H_{i}=\partial T_{i}$ and suppose $\Phi: T_{1} \rightarrow T_{2}$ is a diffeomorphism which induces the identity on $\pi_{1}(K)$. Let $\phi=\Phi \mid H_{1}: H_{1} \rightarrow H_{2}$ and set

$$
V=\overline{M_{1}-T_{1}} \cup \cup_{\phi} \overline{M_{2}-T_{2}} .
$$

Let $\chi\left(M_{2}\right)$ be the result of a surgery on $M_{2}$ along disjointly embedded circles in $M_{2}$ homotopic to the images of the $S_{\alpha}^{1}$. (The framings to be used for this surgery are described in the proof.)

Then if $\pi_{1}\left(K_{1}\right) \rightarrow \pi_{1}\left(M_{1}\right)$ is trivial, $V$ is diffeomorphic to $M_{1} \#\left(\varepsilon \chi\left(M_{2}\right)\right)$ where $\varepsilon= \pm 1$ depending on orientations.

Furthermore: (1) If $M_{1}, M_{2}$ are oriented with $H_{1}, H_{2}$ having induced orientation and $\phi$ is orientation preserving then $\varepsilon=-1$. Otherwise $\varepsilon=+1$.

(2) If, in addition, $\pi_{1}\left(K_{2}\right) \rightarrow \pi_{1}\left(M_{2}\right)$ is trivial then $\chi\left(M_{2}\right)=M_{2} \# W$ where $W=n\left(S^{2} \times S^{2}\right)$ or $W=n(P \# Q)$.

Proof. Since $K_{1}$ is homotopic to zero and of codimension 3 we may without loss of generality [W1], [RS] suppose $K_{1}$ is embedded in some 2-disc $D_{\alpha}$ lying in $M_{1}$.

Then it is clear that we can find 2-discs $D_{\alpha}$ in $M, \alpha=1, \ldots, n$, with disjoint interiors satisfying (i) $D_{\alpha} \cap K_{1}=\partial D_{\alpha}=\operatorname{Im}\left(S_{\alpha}^{1} \hookrightarrow M_{1}\right)$, (ii) $K_{1} \cup$ ( $\cup_{\alpha} D_{\alpha}$ ) collapses to a point in $M_{1}$, and (iii) $\cup_{\alpha} D_{\alpha}$ intersects $H_{1}$ transversely in $n$ disjoint 1-spheres. Set $B_{\alpha}=\overline{T\left(D_{\alpha}\right)-T\left(D_{\alpha}\right) \cap T_{1}}$, (where $T(X)$ will denote a small tubular neighborhood of $X \subset M)$. Then

$$
T_{1} \cup\left(\bigcup_{\alpha} B_{\alpha}\right) \approx T\left(K_{1} \cup\left(\bigcup_{\alpha} D_{\alpha}\right)\right) \approx T(\mathrm{pt})=4 \text {-ball. }
$$

Set $D=T_{1} \cup\left(\cup_{\alpha} B_{\alpha}\right)$ so that

$$
\overline{M_{1}-T_{1}-\bigcup_{\alpha} B_{\alpha}}=\overline{M_{1}-D} \text {. }
$$

Now set

$$
N_{\alpha}=B_{\alpha} \cap H_{1} \quad \text { and } \quad D^{\prime}=T_{2} \cup_{\phi^{-1} \mid \phi\left(\cup_{\alpha} N_{\alpha}\right)}\left(\bigcup_{\alpha} B_{\alpha}\right)
$$

Then clearly

$$
D^{\prime}=\Phi\left(T_{1}\right) \cup_{\phi^{-1} \mid \phi\left(\cup_{\alpha} N_{\alpha}\right)}\left(\bigcup_{\alpha} B_{\alpha}\right)=\tilde{\Phi}\left(T_{1} \cup\left(\bigcup_{\alpha} B_{\alpha}\right)\right)=\tilde{\Phi}(D),
$$

where $\tilde{\Phi}$ is a diffeomorphism obtained by extending $\Phi$ by the identity on $\cup B_{\alpha}$. Clearly then $D^{\prime}$ is a 4-ball.

Now identify $B_{\alpha}$ with $\left(D^{2} \times D^{2}\right)_{\alpha}$ and note that this induces a framing $\phi_{\alpha} \mid\left(S^{1} \times D^{2}\right)_{\alpha} \rightarrow N_{\alpha}$. 
Extend each $\phi_{\alpha}$ to a diffeomorphism $\omega_{\alpha}:\left(S^{1} \times D^{3}\right)_{\alpha} \rightarrow T_{1}$ by 'pushing' $N_{\alpha}$ 'into' $T_{1}$ slightly, while keeping the various $\operatorname{Im} \omega_{\alpha}$ disjoint. Set $\tilde{\omega}_{\alpha}=\Phi \circ \omega_{\alpha}$ and $e_{\alpha}=\dot{\omega}_{\alpha}\left(S^{1} \times 0\right)_{\alpha}$. Note that since $\Phi$ is the identity on $\pi_{1}(K)$ we see that $e_{\alpha}$ is homotopic to $\operatorname{Im}\left(S_{\alpha}^{1} \hookrightarrow M_{2}\right)$.

Then we surger $M_{2}$ along the $e_{\alpha}$ with framing $\tilde{\omega}_{\alpha}$ to obtain $\chi\left(M_{2}\right)$.

But

$$
\begin{aligned}
V & =\overline{M_{1}-T_{1}} \cup_{\phi} \overline{M_{2}-T_{2}} \\
& =\overline{M_{1}-T_{1}-\bigcup_{\alpha} B_{\alpha}} \cup_{\phi} \overline{M_{2}-T_{2} \cup_{\phi^{\prime}}\left(\bigcup_{\alpha} B_{\alpha}\right)}
\end{aligned}
$$

where

$$
\phi^{\prime}=\phi^{-1} \mid \phi\left(\bigcup_{\alpha} N_{\alpha}\right)
$$

and applying Lemma 2.1 we obtain

$$
V=\overline{M_{1}-D} \cup_{\phi} \overline{\chi(M)-D^{1}}=M_{1} \#(\varepsilon \chi(M))
$$

with $\varepsilon$ depending on the orientation conditions in (1).

We extract the case of simply-connected $M_{i}$ as a theorem.

THEOREM 2.3. Suppose $M_{1}, M_{2}$ are oriented simply-connected compact 4 manifolds and let $K_{i} \subset M_{i}$ be the image of an embedding of $\bigvee_{\alpha=1}^{n} S_{\alpha}^{1}$ in $M_{i}$. Let $T_{i}$ be a regular neighborhood of $K_{i}$ and set $H_{i}=\partial T_{i}$. Suppose $\Phi: T_{1} \rightarrow T_{2}$ is a diffeomorphism which induces the identity on $\pi_{1}(K)$ and such that $\phi=\Phi \mid H_{1}$ : $H_{1} \rightarrow H_{2}$ is orientation-reversing relative to the induced orientations on the $H_{i}$.

Let $V=\overline{M_{1}-T_{1}} \cup_{\phi} \overline{M_{2}-T_{2}}$.

Then:

(a) $V$ is simply connected and diffeomorphic to either $M_{1} \# M_{2} \# n\left(S^{2} \times\right.$ $S^{2}$ ) or to $M_{1} \# M_{2} \# n(P \# Q)$.

(b) If $M_{1} \# M_{2}$ has nonzero 2 nd Steifel-Whitney class (i.e. is not spin) then $V \approx M_{1} \# M_{2} \# n(P \# Q)$.

3. Connected sums along 2-manifolds. We now consider 4-manifolds joined along tubular neighborhoods of 2-submanifolds. We recall that if $S$ is a 2-submanifold of a 4-manifold $M$ with tubular neighborhood $T$ in $M$ and if $S^{*}=S-$ open 2-disc then $S^{*}$ has a wedge of 1-spheres $K \subset M$ as deformation retract and $\left.T\right|_{S^{*}}=T^{*}$ is a regular neighborhood of $K$ in $M$.

To facilitate the statement of successive statements we introduce the following definition.

Definition 3.1. Let $i=1,2$. Let $S$ be a 2-manifold and suppose $S_{i}$ is the image of $S$ under an embedding $S \hookrightarrow M_{i}$ into a 4-manifold $M_{i}$. Let $T_{i}$ be a tubular neighborhood of $S_{i}$ in $M_{i}$. Let $H_{i}=\partial T_{i}$. 
Then we say $\phi: H_{1} \rightarrow H_{2}$ is an identity-like diffeomorphism iff there exists a diffeomorphism $\Phi: T_{1} \rightarrow T_{2}$ which induces the identity on $\pi_{1}(S)$ such that (i) there exist 2-discs $d_{i} \subset S_{i}$ such that $\Phi\left|T_{1}\right|_{d_{1}}:\left.\left.T_{1}\right|_{d_{1}} \rightarrow T_{2}\right|_{d_{2}}$ is a fiber-preserving diffeomorphism and (ii) $\phi=\Phi \mid H_{1}$.

By a slight abuse of notation if $S$ is simply a l-complex and $T_{i}$ is a regular neighborhood of $S_{i}$ in $M_{i}$ we will continue to say $\phi$ is an identity-like diffeomorphism provided it is simply the restriction of a diffeomorphism $\Phi$ : $T_{1} \rightarrow T_{2}$ inducing the identity on $\pi_{1}(S)$.

Our main point of interest will be diffeomorphisms $\phi: H_{1} \rightarrow H_{2}$ which are fiber-bundle maps of the $H_{i}$ as $S^{1}$-bundles. Clearly such maps will always be identity-like. Furthermore, if $d_{i}$ is a disc in $S_{i}$ with $\phi \mid H_{1 \mid d_{1}}: H_{1 \mid d_{1}} \rightarrow H_{2 \mid d_{2}}$ a diffeomorphism, then $\phi\left|H_{1}\right| \overline{S_{1}-d_{1}}$ always extends to an identity-like diffeomorphism $\tilde{\phi}: H_{1} \rightarrow H_{2}$, where $H_{i}^{*}=\partial\left(T_{i} \mid \overline{S_{i}-d_{i}}\right)$, and we think of $T_{i} \mid \overline{S_{i}-d_{i}}$ as a regular neighborhood of an embedded 1-complex $K_{i}=$ image of the 1-skeleton of ( $S$-disc).

We can now state

LEMMA 3.2. Suppose $M_{1}, M_{2}^{\prime}$ are compact 4-manifolds with compact 2submanifolds $S_{1}, S_{2}^{\prime}$, respectively. Let $T_{1}, T_{2}^{\prime}$ be tubular neighborhoods of $S_{1}, S_{2}^{\prime}$ with projection maps $\pi, \pi^{\prime}$ and set $H_{1}=\partial T_{1}, H_{2}^{\prime}=\partial T_{2}^{\prime}$.

Suppose $\eta: H_{1} \rightarrow H_{2}$ is an identity-like diffeomorphism and $V=\overline{M_{1}-T_{1}} \cup_{\eta} \overline{M_{2}^{\prime}-T_{2}^{\prime}}$. Suppose also some fiber $C$ of $H_{1}$ considered as a loop in $V$ is homotopic to zero in $V$. Then there exist 2-discs $d_{1}$ in $S_{1}$ and $d_{2}$ in $S_{2}^{\prime}$ such that if $S_{1}^{*}=\overline{S_{1}-d_{1}}, S_{2}^{\prime *}=\overline{S_{2}^{\prime}-d_{2}}, \tilde{H}_{1}=\left.H_{1}\right|_{S_{i}^{*}}, \tilde{H}_{2}^{\prime}=\left.H_{2}^{\prime}\right|_{S_{2}^{*}}, T_{1}^{*}=$ $\left.T_{1}\right|_{S_{1}^{*}}, \quad T_{2}^{\prime *}=\left.T_{2}\right|_{S_{2}^{*}}, \quad H_{1}^{*}=\partial T_{1}^{*}$ and $H_{2}^{\prime *}=\partial T_{2}^{\prime *}$ then $\tilde{\eta}=\eta \mid H_{1}$ is a diffeomorphism of $\tilde{H}_{1}$ and $\tilde{H}_{2}^{\prime}$ which extends to an identity-like diffeomorphism $\eta^{*}: H_{1}^{*} \rightarrow H_{2}^{\prime *}$ such that if

$$
V^{\prime}=\overline{M_{1}-T_{1}^{*}} \cup_{\eta^{*}} \overline{M_{2}^{\prime}-T_{2}^{\prime *}}
$$

then $V^{\prime}$ is diffeomorphic to either $V \#(P \# Q)$ or to $V \#\left(S^{2} \times S^{2}\right)$.

Proof. Let $\pi(C)=p \in S, C^{\prime}=\eta(C)$ is a fiber of $H^{\prime}$ and we can without loss of generality suppose that for some discs $d_{1} \ni p$ in $S_{1}$ and $d_{2}^{\prime} \ni p^{\prime}=$ $\pi^{\prime}\left(C^{\prime}\right) \in S^{\prime}$ we have that $\eta \mid \tilde{H}_{1}$ is a diffeomorphism of $\tilde{H}_{1}$ onto $\tilde{H}_{2}^{\prime}$ which extends to an identity-like diffeomorphism $\eta^{*}$ of $H_{1}^{*}$ onto $H_{2}^{* *}$. Let $N_{1}=$ $\pi^{-1}\left(d_{1}\right)$ and $N_{2}=\left(\pi^{\prime}\right)^{-1}\left(d_{2}\right)$. Push $N_{1}$, respectively $N_{2}$, 'into' $\overline{M_{1}-T_{1}}$, respectively $\overline{M_{2}^{\prime}-T_{2}^{\prime}}$, using some inward pointing vector field to get submanifolds $\tilde{N}_{1} \approx\left(S^{1} \times D^{2}\right) \times I, \quad \tilde{N}_{2} \approx\left(S^{\prime} \times D^{2}\right) \times I$ of $\overline{M_{1}-T_{1}}$, respectively $\overline{M_{2}^{\prime}-T_{2}^{\prime}}$. Note that $N_{1} \subset \partial \tilde{N}_{1}$ and $N_{2} \subset \partial \tilde{N}_{2}$ and using $\eta$ we can identify $N_{1}$ with $N_{2}$ in $V$ to obtain a neighborhood $N(C)=\tilde{N}_{1} \cup_{N_{1}=\eta\left(N_{2}\right)} \tilde{N}_{2}$ of $C$ in $V$. Note that $N(C) \approx C \times D^{3}$. Now surger $V$ along $N(C)$ using the induced framing of $C$ as a fiber of $H$ to obtain $\chi(V)=\overline{V-N(C)} \cup_{f} D^{2} \times$ 
$S^{2}$ where $f: \partial N(C) \rightarrow S^{1} \times S^{2}$ is the identifying diffeomorphism along the boundary.

Let $f_{1}=\overline{f \mid \partial \tilde{N}_{1}-N_{1}}$ and $f_{2}=\overline{f \mid \partial \tilde{N}_{2}-N_{2}}$. Then we can write

$$
\chi(V)=\overline{M_{1}-T_{1}-N_{1}} \cup_{f_{1}} D^{2} \times S_{+}^{2} \cup_{\eta} \overline{M_{2}^{\prime}-T_{2}^{\prime}-\tilde{N}_{2}} \cup_{f_{2}} D^{2} \times S_{-}^{2} .
$$

But

and

$$
\overline{M_{1}-T_{1}-\tilde{N}_{1}} \cup_{f_{1}} D^{2} \times S_{+}^{2} \simeq M_{1}-T_{1} \cup_{\tilde{f}_{1}} D^{2} \times D^{2}
$$

$$
\overline{M_{2}^{\prime}-T_{2}^{\prime}-\tilde{N}_{1}} \cup_{f_{1}} D^{2} \times S_{-}^{2} \simeq \overline{M_{2}^{\prime}-T_{1}^{\prime}} \cup_{\tilde{f}_{2}} D^{2} \times D^{2}
$$

where $\tilde{f}_{1}, \tilde{f}_{2}$ are diffeomorphisms of $N_{1}$, respectively $N_{2}$, onto $S^{\mathrm{r}} \times D^{2}$ induced by $f_{1}, f_{2}$.

However the framing on $D^{2} \times S^{1}$ was induced by the framing of $C$ as a fiber so that we can conclude that

$$
\overline{M_{1}-T_{1}} \cup_{\tilde{f}_{1}} D^{2} \times D^{2} \approx \overline{M_{1}-T_{1}} \mid \overline{S_{1}-d_{1}}=\overline{M_{1}-T_{1}^{*}}
$$

and similarly

$$
\overline{M_{2}^{\prime}-T_{2}^{\prime}} \cup \tilde{f}_{2} D^{2} \times D^{2} \approx \overline{M_{2}^{\prime}-T_{2}^{\prime *}} .
$$

Thus $\chi(V) \approx V^{\prime}$. But $C$ is homotopic to zero in $V$. Thus by Theorem $\mathrm{W}$ we have that $\chi(V)$ is either $V \#\left(S^{2} \times S^{2}\right)$ or $V \#(P \# Q)$ and so our theorem is proven.

In almost all cases of interest when we encounter manifolds $V$ admitting the above decomposition it turns out that $M_{2}^{\prime}$ arises from some manifold $M_{2}$ by means of a $\sigma$-process at some point on a compact 2-submanifold $S_{2} \subset M_{2}$. We then have

LeMma 3.3. Suppose $M_{1}, M_{2}, M_{2}^{\prime}$ are compact 4-manifolds containing 2submanifolds $S_{1}, S_{2}, S_{2}^{\prime}$, respectively. Suppose $p \in S_{2} \subset M_{2}$ and $\sigma: M_{2}^{\prime} \rightarrow M_{2}$ (resp. $\bar{\sigma}: M_{2}^{\prime} \rightarrow M_{2}$ ) is a smooth map of $M_{2}^{\prime}$ onto $M_{2}$ such that (i) $L=\sigma^{-1}(p)$ is an embedded 2-sphere in $M_{2}^{\prime}$ and $L \cdot L=-1$ (resp. $L=\bar{\sigma}^{-1}(p)$ an embedded 2-sphere with $L \cdot L=+1$ ), (ii) $\sigma$ (resp. $\sigma$ ) restricts to a diffeomorphism of $M_{2}^{\prime}-L$ onto $M_{2}-p$ and $S_{2}^{\prime}$ onto $S_{2}$. (The above hypotheses on $\sigma$ are equivalent to assuming that $\sigma: M_{2}^{\prime} \rightarrow M_{2}$ is $M_{2}$ blown up by a $\sigma$-process at $p \in S_{2} \subset M_{2}$ and $S_{2}^{\prime}$ is the strict image of $S_{2}$ in $M_{2}^{\prime}$.) Let $T_{1}, T_{2}, T_{2}^{\prime}$ be tubular neighborhoods of $S_{1}, S_{2}, S_{2}^{\prime}$, respectively, and set $H_{1}=\partial T_{1}, H_{2}=\partial T_{2}, H_{2}^{\prime}$ $=\partial T_{2}^{\prime}$. Suppose $\eta: H_{1} \rightarrow H_{2}^{\prime}$ is identity-like and that $V=\overline{M_{1}-T_{1}} \cup_{\eta} \overline{M_{2}^{\prime}-T_{2}^{\prime}}$. Then there exist a 2-disc $d_{2}$ about $p$ in $S_{2}$ and $a$ 2-disc $d_{1}$ in $S_{1}$, such that, denoting $S_{1}^{*}=\overline{S_{1}-d_{1}}, S_{2}^{*}=\overline{S_{2}-d_{2}}, T_{i}^{*}=\left.T_{i}\right|_{S_{i}^{*}}$, $H_{i}^{*}=\partial T_{i}^{*}, \tilde{H}_{i}=\left.H_{i}\right|_{s_{i}^{*}} \eta \mid \tilde{H}_{1}$ is a diffeomorphism of $\tilde{H}_{1}$ onto $\tilde{H}_{2}$ which extends to an identity-like diffeomorphism $\eta^{*}: H_{1}^{*} \rightarrow H_{2}^{*}$, such that if $V^{\#}=\overline{M_{1}-T_{1}^{*}} \cup_{\eta^{*}} \overline{M_{2}-T_{2}^{*}}$, then $V^{\#}$ is diffeomorphic to $V \# P$ (respectively, $V \# Q$ ). 
Furthermore, if $F$ is a fiber of $H_{1}$ in $V$ then $F$ is homotopic to zero in $V$.

REMARK. We shall indicate two proofs of the lemma. First we shall show how Lemma 3.3 is a consequence of Lemma 3.2. We then shall sketch an independent proof of Lemma 3.3 not involving Lemma 3.2 or surgery theory but rather more heavily algebraic-geometric.

Proof. (1) Let $p^{\prime}$ be the unique point in $S_{2}^{\prime} \cap L$. Without loss of generality we can suppose that $\overline{L \cap T_{2}^{\prime}}$ is just the fiber of $T_{2}^{\prime}$ over $p^{\prime}$. Let $C^{\prime}=\overline{L \cap H_{2}^{\prime}}$. Without loss of generality we may also suppose that $C=\eta^{-1}\left(C^{\prime}\right)$ is a fiber of $H_{1}$ over some point $q \in S_{1}$. Now $L^{*}=\overline{L \cap M_{2}^{\prime}-T_{2}^{\prime}}$ can be considered as an embedded 2-disc in $V$ bounding $C$ so that $C$ is homotopic to zero in $V$. We

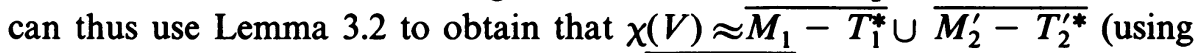
the notations of Lemma 3.2). But $L \subset \overline{M_{2}^{\prime}-T_{2}^{* \prime}}$ by construction so that $L \subset \chi(V)$. Now $L$ is an embedded 2-sphere with self-intersection -1 (resp. $+1)$. Thus if $N$ is a tubular neighborhood of $L$ we can easily verify that $\partial N=S^{3}$ and $N \approx Q-\{4$-ball $\}$. Taking $N$ sufficiently small we note that

$$
\chi(V)-N \approx \overline{M_{1}-T_{1}} \cup \overline{\left(M_{2}^{\prime}-T_{2}^{\prime}-N\right)}
$$

and

$$
M_{2}^{\prime}-T_{2}^{\prime}-N \approx M_{2}^{\prime}-T_{2}^{*}-\{4 \text {-ball }\} .
$$

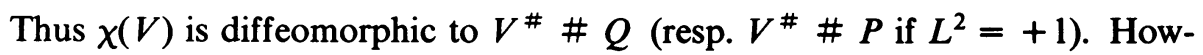
ever using Lemma 3.2 and noting that $L^{2} \not 0(\bmod 2)$ we see that $\chi(V) \approx$ $V \# P \# Q$. In addition, analyzing the surgery we performed on $C$ in Lemma 3.2 we can obtain that our tubular neighborhood $N$ above can be identified with the factor $\# Q$ in $V \# P \# Q$. (Resp. the factor \#P if $L^{2}=+1$.) Thus splitting off $N$ we find $V^{\#} \approx V \# P$ (resp. $V^{\#} \approx V \# Q$ if $\left.L^{2}=+1\right)$.

(2) Let $p, p^{\prime}, L$ be as in (1). Let $\zeta \in L-\overline{L \cap T_{2}^{\prime}} \subset V$ and blow up $B$ at $\zeta$ by a $\bar{\sigma}$-process. Let $M_{2}^{\prime \prime}$ denote $M_{2}^{\prime}$ blown up at $\zeta$ and let $A^{\prime \prime}$ be the strict image of any $A^{\prime} \subset M_{2}^{\prime}$. We thus have that

$$
V \# P \approx \overline{M_{1}-T_{1}} \cup_{\eta^{\prime}} \overline{M_{2}^{\prime \prime}-T_{2}^{\prime \prime}},
$$

where $\eta^{\prime}$ is the obvious induced diffeomorphism. We note that $L^{\prime}$, the strict image of $L$ in $M_{2}^{\prime \prime}$, is a sphere with $L^{\prime} \cdot L^{\prime}=0$. Thus we can find a disc $d$ about $p^{\prime \prime} \in S_{2}^{\prime \prime}$ and a collection $G \approx d \times L^{\prime}$ of 2-spheres in $M_{2}^{\prime \prime}$ such that $G \cap S_{2}^{\prime \prime}=d$ and $G \cap T_{2}^{\prime \prime}=\left(\pi_{2}^{\prime \prime}\right)^{-1}(d)$ where $\pi_{2}^{\prime \prime}: T_{2}^{\prime \prime} \rightarrow S_{2}^{\prime \prime}$ is the obvious projection map. Let $B=\overline{G-G \cap T_{2}^{\prime \prime}}$ so that $B \approx D^{2} \times D^{2}$ with $B \cap H_{2}^{\prime \prime}$ $=\partial B \cap H_{2}^{\prime} \approx S^{1} \times D^{2}$.

We can write

$$
V \# P \approx \overline{M_{1}-T_{1} \bigcup_{\eta_{1}^{\prime}} B} \cup_{\tilde{\eta}^{\prime}} \overline{M_{2}^{\prime \prime}-T_{2}^{\prime \prime}-B}
$$


where $\eta_{1}^{\prime}=\eta^{\prime} \mid \eta^{\prime-1}\left(B \cap H_{2}^{\prime \prime}\right)$ and $\tilde{\eta}^{\prime}=\eta^{\prime} \mid H_{1}-\eta^{\prime-1}\left(B \cap H_{2}^{\prime \prime}\right)$. It is clear that $\overline{M_{1}-T_{1} \cup_{\eta_{1}^{\prime}} B} \approx \overline{M_{1}-T_{1}^{*}}$ and to conclude our proof it suffices to show that

$$
\overline{M_{2}^{\prime \prime}-T_{2}^{\prime \prime}-B} \approx \overline{M_{2}-T_{2}^{*}}
$$

(where $d_{2}$ is the image of some disc $D$ in $S_{2}$ and $d_{1}$ is obtained in the obvious fashion in $S_{1}$ and $T_{1}^{*}, T_{2}^{*}$ are defined accordingly).

To demonstrate (*) first denote the preimage of $\zeta$ in $M_{2}^{\prime \prime}$ by $\Gamma$ and let $Z$ be a regular neighborhood of the wedge of 2 -spheres $L^{\prime}+\Gamma$ in $M_{2}^{\prime \prime}$. Let $D=Z \cap S_{2}^{\prime \prime}$. Thus $D$ is a disc on $S_{2}^{\prime \prime}$ about $p^{\prime \prime}$ and we may assume without loss of generality that $D=d$ and that $Z \cap T_{2}^{\prime \prime}=G \cap T_{2}^{\prime \prime}$.

Then it is readily verifiable that

$$
T_{2}^{\prime \prime} \cap\left(M_{2}^{\prime \prime}-Z\right) \approx T_{2}^{\prime \prime} \mid \overline{S_{2}^{\prime \prime}-D}=T_{2}^{\prime \prime *}
$$

and since $B \subset Z$ if $d_{2}$ is the image of $D$ in $M$ then

$$
\overline{M_{2}^{\prime \prime}-T_{2}^{\prime \prime}-B} \approx \overline{M_{2}-T_{2}\left|\overline{S_{2}-d}-T_{2}\right|_{d}} \bigcup_{\partial\left(T_{2} \mid d\right)=\partial Z} \overline{Z-T_{2}^{\prime \prime} \cap Z-B}
$$

where the identification is that induced by the identification of $\partial\left(T_{2} \mid d\right)$ with $\partial Z \approx S^{3}$.

It is thus sufficient to show that $\overline{Z-T_{2}^{\prime \prime} \cap Z-B}$ is a 4-ball to conclude that $M_{2}^{\prime \prime}-T_{2}^{\prime \prime}-B \approx M_{1}-T_{2}^{*}$ where $T_{2}^{*}=\left.T_{2}\right|_{\overline{S_{2}-d_{2}}}$. But

$$
Z-T_{2}^{\prime \prime} \cap Z-B=Z-G \text {. }
$$

But $Z$ is just a regular neighborhood of the wedge of 2-spheres $L+\Gamma$ and so $Z \approx P \# Q-R$ where $R$ is a 4-ball. $G$ is just a tubular neighborhood of a fiber of $Z$. We can thus write $Z-G \approx S^{2} \times D^{2}-R$. Let $\rho: S^{2} \times D^{2} \rightarrow D^{2}$ be the projection map. Then it is seen that

$$
\begin{aligned}
R & \cap \partial\left(S^{2} \times D^{2}\right)=\partial R \cap \partial\left(S^{2} \times D^{2}\right) \\
& =\text { tubular neighborhood of a cross-section of } \partial\left(S^{2} \times D^{2}\right) \stackrel{\rho \mid \partial\left(S^{2} \times D^{2}\right)}{\rightarrow} S^{1} .
\end{aligned}
$$

But then by Sublemma 3.3a below $Z-G$ is a 4-ball as desired.

Sublemma 3.3a (Compare [MM1]). Suppose $\pi: S^{2} \times D^{2} \rightarrow D^{2}$ is the obvious projection and $\rho: S^{2} \times S^{1} \rightarrow S^{1}$ is just $\pi \mid S^{2} \times S^{1}$. Let $B$ be a 4-ball embedded in $S^{2} \times D^{2}$ such that

$$
R=B \cap \partial\left(S^{2} \times D^{2}\right)=B \cap\left(S^{2} \times S^{1}\right)=\partial B \cap\left(S^{2} \times S^{1}\right)
$$

is a tubular neighborhood in $S^{2} \times S^{1}$ of a cross-section of $\rho$.

Then $\overline{S^{2} \times D^{2}-B}$ is a 4-ball.

Proof. Let $Y=\overline{S^{2} \times D^{2}-B}$. Let $W=D^{3} \times S^{1}$ and attach $W$ to $S^{2} \times$ $D^{2}$ above so as to get $S^{2} \times D^{2} \cup W \approx S^{\prime \prime}$. Note that $R \subset \partial\left(S^{2} \times D^{2}\right)$ $=\partial W$ and that $W \approx R \times I$. Thus since $R \subset \partial B$ we have $B \cup W \approx B$. But 
then

$$
Y \approx \overline{S^{2} \times D^{2} \cup W-B \cup W} \approx \overline{S^{4}-(B \cup W)} \approx \overline{S^{4}-B} .
$$

But it is well known (see [H], [RS] for example) that $\overline{S^{4}-B}$ is a 4-ball.

Combining the results of $\$ 2$ with the above lemmas we are led to

THEOREM 3.4. Suppose $M_{1}, M_{2}^{\prime}$ are oriented compact 4-manifolds and suppose $S_{1}, S_{2}^{\prime}$ are compact 2-submanifolds of $M_{1}, M_{2}^{\prime}$, respectively, with tubular neighborhoods $T_{1}, T_{2}^{\prime}$, respectively. Set $H_{1}=\partial T_{1}, H_{2}^{\prime}=\partial T_{2}^{\prime}$, respectively, and suppose $\eta: H_{1} \rightarrow H_{2}$ is an identity-like orientation reversing diffeomorphism.

Let $V=\overline{M_{1}-T_{1}} \cup_{\eta} \overline{M_{2}^{\prime}-T_{2}^{\prime}}$. Suppose $K_{1}, K_{2}$ are 1-skeletons of $S_{1}, S_{2}^{\prime}$ and $K_{1}, K_{2}$ are homotopic to zero in $M_{1}$, respectively $M_{2}^{\prime}$. Then:

(1) If $C$ is a fiber of $H_{1}$ in $V$ which is homotopic to zero in $V, k=$ $r k H_{1}\left(K_{1}, Z\right)$, then either

(a) $V \#\left(S^{2} \times S^{2}\right)$ is diffeomorphic to $M_{1} \# M_{2}^{\prime} \# k\left(S^{2} \times S^{2}\right)$, or

(b) $V \#(P \# Q)$ is diffeomorphic to $M_{1} \# M_{2}^{\prime} \# k(P \# Q)$.

If either $M_{1}$ or $M_{2}^{\prime}$ is of odd-type then alternatives (a) and (b) both hold.

(2) If $M_{2}^{\prime}$ is obtained by blowing up $M_{2}$ by means of a $\sigma$ or $\bar{\sigma}$ process as in Lemma 2.3, then in the former case

$$
V \# P \approx M_{1} \# M_{2} \# k(P \# Q)
$$

and in the latter

$$
V \# Q \approx M_{1} \# M_{2} \# k(P \# Q) .
$$

Proof. (1) Using Lemma 3.2 and Proposition 2.2, and noting that if $X$ is of odd type with $X \approx W \# S^{2} \times S^{2}$, then also $X \approx W \# P \# Q$, we obtain that either

$$
V \# P \# Q \approx M_{1} \# M_{2}^{\prime} \# k(P \# Q)
$$

or

$$
V \#\left(S^{2} \times S^{2}\right) \approx M_{1} \# M_{2}^{\prime} \# k\left(S^{2} \times S^{2}\right) .
$$

Clearly if $M_{1} \# M_{2}^{\prime}$ is of odd type the first alternative above will hold.

(2) If $M_{2}^{\prime}$ is obtained by a blowing up we can apply Lemma 3.3 and Proposition 2.2 to obtain the desired result.

We extract a particularly simple case of our theorem for separate mention.

CoROllary 3.5. Suppose $M_{1}, M_{2}$ are oriented simply-connected compact 4-manifolds and $S_{1}, S_{2}$ are oriented compact 2-submanifolds of genus g. Let $M_{2}^{\prime}$ be $M_{2}$ blown up by a $\sigma$-process at some point of $S_{2}$ and denote the strict image of $S_{2}$ in $M_{2}^{\prime}$ by $S_{2}^{\prime}$. Let $T_{1}$, $T_{2}^{\prime}$ be tubular neighborhoods of $S_{1}, S_{2}^{\prime}$ in $M_{1}, M_{2}^{\prime}$, respectively, and set $H_{1}=\partial T_{1}, H_{2}^{\prime}=\partial T_{2}^{\prime}$.

Suppose $\eta: H_{1} \rightarrow H_{2}^{\prime}$ is a fiber bundle diffeomorphism of $H_{1}$ onto $H_{2}^{\prime}$ reversing orientation. 
Let $V=\overline{M_{1}-T_{1}} \cup_{\eta} \overline{M_{2}^{\prime}-T_{2}^{\prime}}$. Then $V \# P$ is diffeomorphic to $M_{1} \# M_{2} \# 2 g(P \# Q)$.

In order to apply our results on irrational connected sums to determine the structure of algebraic surfaces we recall two results from our work in [MM2, $\S 2]$.

Theorem 3.6 (See Corollary 2.4 of [MM2]). Let $W$ be a complex manifold. Suppose $f: W \rightarrow \mathbf{C}$ is a nonconstant proper holomorphic mapping of $W$ onto a disc $\Delta$ about the origin such that 0 is a critical value of $f$. Suppose the zero divisor $Z$ of $f$ consists of two nonsingular irreducible components $A_{1}, A_{2}$ of multiplicity 1 crossing normally in a nonsingular irreducible subvariety $S$.

Suppose $\Gamma \rightarrow S$ is a tubular neighborhood of $S$ in $W$ such that $T_{1}=\Gamma \cap A_{1}$ and $T_{2}=\Gamma \cap A_{2}$ are tubular neighborhoods of $S$ in $A_{1}$, respectively $A_{2}$. Set $H_{i}=\partial T_{i} \rightarrow S$.

Then there exists an orientation-reversing bundle isomorphism $\eta: \mathrm{H}_{1} \rightarrow \mathrm{H}_{2}$ such that for any regular value $\lambda \in \Delta$ of $f$ if $V_{\lambda}=f^{-1}(\lambda)$ then

$$
V \approx \overline{A_{1}-T_{1}} \cup_{\eta} \overline{A_{2}-T_{2}} \text {. }
$$

TheOREM 3.7 (See CoRollary 2.5 OF [MM2]). Let $W$ be a compact complex manifold. Suppose $V, X_{1}, X_{2}$ are compact complex submanifolds of $W$ intersecting normally and denote $X_{1} \cap X_{2}$ by $S$ and $V \cap X_{1} \cap X_{2}$ by $C$. Suppose as divisors on $W, V$ is linearly equivalent to $X_{1}+X_{2}$.

Let $\sigma: X_{2}^{\prime} \rightarrow X_{2}$ be the monoidal transformation of $X_{2}$ with center $C$ and let $S^{\prime}$ be the strict image of $S$ in $X_{2}^{\prime}$.

Let $T_{2}^{\prime} \rightarrow S, T_{1} \rightarrow S$ be tubular neighborhoods of $S^{\prime}$ in $X_{2}^{\prime}$ and $S$ in $X_{1}$, respectively, with $H_{2}^{\prime}=\partial T_{2}^{\prime}$ and $H_{1}=\partial T_{1}$.

Then there exists a bundle-isomorphism $\eta: H_{2}^{\prime} \rightarrow H_{1}$ which is orientation reversing such that

$$
V \approx \overline{X_{2}^{\prime}-T_{2}^{\prime}} \cup_{\eta} \overline{X_{1}-T_{1}}
$$

Proof. We can reduce Theorem 3.7 to 3.6 by first blowing up $W$ along $V \cap X_{1}$ and then blowing up the resultant manifold $W^{\prime}$ along the strict image of $V \cap X_{2}$ in $W^{\prime}$. This then separates the strict image of $V$ from that of $X_{1}+X_{2}$ and allows us to apply 3.6. For more details see the above reference. For our work we need a special case of 3.7, combined with 3.5.

Corollary 3.8. Suppose in Theorem 3.7, $W$ is of complex dimension 3 and $V \cdot X_{1} \cdot X_{2}=n>0$. Then if $S$ is connected of genus $g$ and $X_{1}, X_{2}$ are simply connected, we have

$$
V \# P \approx X_{1} \# X_{2} \#(n-1) Q \# 2 g(P \# Q) .
$$


Proof. By 3.7 we have $V \approx \overline{X_{2}^{\prime}-T_{2}^{\prime}} \cup_{\eta} \overline{X_{1}-T_{1}}$. But $C$ in our case is just $n$ distinct points and $X_{2}$ blown up along $C$ is then topologically $X_{2} \# n Q$. Applying Corollary 3.5 we obtain

$$
V \# P \simeq\left(X_{2} \#(n-1) Q\right) \# X_{1} \# 2 g(P \# Q)
$$

as desired.

\section{Applications to algebraic surfaces.}

LEMMA 4.1. Suppose $X$ is a nonsingular algebraic surface which is almost completely decomposable. Let $W \stackrel{\pi}{\rightarrow} X$ be an analytic $C P^{1}$-bundle over $X$ and let $F$ denote a fiber of $W \stackrel{\pi}{\rightarrow} X$.

Suppose $V$ is an irreducible nonsingular subvariety of $W$ with $V \cdot F=k>0$ and suppose there are given divisors $E_{j}$ on $W, j=1, \ldots, k$, such that $E_{j} \cdot F=$ 1 and there exist irreducible nonsingular representatives $V_{i} \in\left|\sum_{j=1}^{i} E_{j}\right|, W_{j} \in$ $\left|E_{j}\right|$ such that:

(1) $V_{i}, V_{i+1}, W_{i+1}$ have normal crossing in $W$ with $V_{i} \cdot V_{i+1} \cdot W_{i+1} \neq 0$ for $i=1, \ldots, k-1$.

(2) $V_{i} \cap W_{i+1}$ has genus greater than zero or either $W_{i+1}$ is rational or $V_{i}$ is rational. Then if $V \in\left|D_{k}\right|$ then $V$ is almost completely decomposable.

Proof. We use induction on $k=V \cdot F$. If $k=1$ then $\pi \mid V$ is a morphism of degree 1 of $V$ onto $X$. Thus $V$ is simply $X$ blown up at a finite number $m$ of points and so $V \approx X \# m Q$. Thus since $X$ was A.C.D. so is $V$. Now suppose the lemma is true whenever $V \cdot F \leqslant l-1$ and suppose that $V \cdot F=l>1$.

Set $D_{i}=\Sigma_{1}^{i} E_{j}$ and let $V_{l-1}, V_{l}, W_{l}$ be irreducible nonsingular elements of $\left|D_{l-1}\right|,\left|D_{l}\right|,\left|E_{l}\right|$, respectively, satisfying the hypothesis of our lemma. Let $t=V_{l-1} \cdot V_{l} \cdot W_{l}>0$ and $g=$ genus $\left(V_{l-1} \cap W_{l}\right) \geqslant 0$.

Then by Corollary 3.8 we have that

$$
V_{l} \# P \approx V_{l-1} \# W_{l} \#(t-1) Q \# 2 g(P \# Q) \text {. }
$$

Suppose $g>0$. Then by our inductive hypothesis we find $V_{l-1}, W$ are A.C.D. and since $g>0$ the right side of $(*)$ is completely decomposable. Thus $V$ is A.C.D. If $g=0$ then either $V_{l-1}$ or $W_{l}$ is rational. However since the left side of (*) is of odd type we can conclude that the right side is diffeomorphic to either $V_{l-1} \# P \#(v+t-1) Q$ or to $W_{l} \# P \#(w+t$ $-1) Q$ for some integers $v, w$. In either case use of the inductive hypothesis shows that $V_{l}$ is A.C.D. Since $V$ is linearly equivalent to $V_{l}$ and both are nonsingular they are diffeomorphic. Thus $V$ is A.C.D. as desired.

THEOREM 4.2. Suppose $X$ is an almost completely decomposable nonsingular algebraic surface. Let $C$ be some irreducible nonsingular hypersurface section of $X$ in some embedding $X \hookrightarrow C P^{N}$. Let $E=[k C]$ be the line bundle over $X$ associated to the divisor $k C, k \geqslant 0$, and let $W \stackrel{\pi}{\rightarrow} X$ be the projective closure of 
$E \rightarrow X$ so that $W$ is a $C P^{1}$-bundle over $X$. Let $S=\pi^{-1}(C) \subset W$ and let $X \hookrightarrow W$ also denote $X$ embedded as the 'zero-section' of $W$.

Then for any $m>0$ and $l>0$ there exists an irreducible nonsingular $V \in|m X+l S|$. If $V_{(m, l)}$ is any irreducible nonsingular element of $|m X+l S|$ then $V_{(m, l)}$ is almost completely decomposable.

Furthermore if $k \neq 0$ then the above conclusion is also true for $l=0$.

Proof. Let $H_{c}$ be a hypersurface of $C P^{N}$ cutting out $C \subset X$ and let $d=\operatorname{deg} H_{c}$. Now fix $m>0$ and $l \geqslant 0$ and let $H_{m k+l}, J_{l}$ be hypersurfaces of degree $d(m k+l)$, $d l$, respectively, cutting out nonsingular hypersurface sections $C_{m k+l}, S_{l}$ of $X$, respectively, such that $C_{m k+l}$ intersects $S_{l}$ transversely in $X$. Clearly using Bertini's theorem we can always produce such $H_{m k+l}, J_{l}$.

Now let $\left\{U_{\alpha}\right\}$ be a coordinate cover of $X$ trivializing $E$ and thus $W$. Let $C_{m k+l}, S$, have local equations $e_{\alpha}=0, d_{\alpha}=0$, respectively, in $U_{\alpha}$ and suppose $W$ has fiber coordinate $\left(\zeta_{0 \alpha}, \zeta_{1 \alpha}\right)$. Let $V_{(m, l)} \subset W$ be the subset of $W$ given locally by $d_{\alpha} \zeta_{1 \alpha}^{m}-e_{\alpha} \zeta_{0 \alpha}^{m}=0$ (where $X \hookrightarrow W$ is given locally by $\zeta_{1 \alpha}=0$ ). Then an explicit computation shows that $V_{(m, l)}$ is a nonsingular subvariety of $W$, irreducible if $l>0$ or $l=0$ and $k>0$. Furthermore we find $V_{(m, l)} \cdot F=m$ and $V_{(m, l)} \cdot C=(k m+l) C^{2}$ so that $V_{(m, l)} \in|m X+l S|$.

Now suppose $l>0$ or $l=0$ and $k>0$. Let $V \in|m X+l S|$ be an irreducible nonsingular subvariety of $W$. Let $D_{i}=i X+l S$ for $1 \leqslant i \leqslant m$ and $E_{j}=X$ for $2 \leqslant j \leqslant m$. It is clear by our construction of the $V_{(m, l)}$ above that we can always choose appropriate hypersurfaces in $C P^{N}$ such that there exist irreducible nonsingular $V_{i} \in\left|D_{i}\right|$ and $W_{j} \in\left|E_{j}\right|$ so that $V_{i}, V_{i+1}, W_{i+1}$ cross normally in $W$ for $1 \leqslant i \leqslant m-1$. Furthermore

$$
V_{i} \cdot V_{i+1} \cdot W_{i+1}=i(i+1) X^{3}+2(i+1) l X^{2} S+l^{2} X S^{2}>0 .
$$

Then by the adjunction formula we have that

$$
g_{i}=\operatorname{genus}\left(V_{i} \cap W_{i+1}\right)=\operatorname{genus}\left(r_{i} C\right)=\left(r_{i}\left(r_{i}-1\right) C^{2}-r_{i} \chi_{0}\right) / 2
$$

where $\chi_{0}=2-2 g(C)$ and $r_{i}=k i+l$. Then if $g(C)>0$ we have $g_{i}>0$ all $i$. Suppose $g(C)=0$. Then $X$ has a rational hypersurface section and so must be rational. But $W_{j}$ is linearly equivalent to $X$ for all $j$ and so is also rational.

We can thus apply Lemma 4.1 to conclude that $V$ is A.C.D.

We can apply our theorem to analytic or branched covers (see [GR,III.B], a branched cover is an analytic cover with nonempty critical set).

So suppose $V \stackrel{\pi}{\rightarrow} X$ is as above with $k>0$. Let $V$ be a nonsingular irreducible subvariety of $W$ such that $\pi \mid V$ exhibits $V$ as an $m$-fold branched cover of $X$. Thus as a cycle in $W$ we have $V \sim m X+Z$ where $Z \in \rho\left(H_{2}(X)\right)$ in the natural splitting $H_{4}(W) \approx \rho\left(H_{2}(X)\right) \oplus H_{4}(X)$ induced by the portion

$$
0 \rightarrow H_{2}(X) \stackrel{\rho}{\rightarrow} H_{4}(W) \stackrel{\pi}{\rightarrow} H_{4}(X) \rightarrow 0
$$


of the Thom-Gysin sequence of the 2-sphere bundle $W \rightarrow X$. If $V \sim m X$ we will call $V$ a pure $m$-fold branched cover. But in this case $V \sim V_{(m, 0)}$ and so is A.C.D. by Theorem 4.2. We thus have

COROLlaRY 4.3. Suppose $V$ is a nonsingular subvariety of $W$ above, with $\pi$ : $V \rightarrow X$ a pure $m$-fold branched cover of $X$.

Then $V$ is A.C.D.

We recall the following definition [Wk, Definition 15].

Definition 4.4. Let $(M, \pi, V)$ be a $k$-sheeted branched covering of $V$ where $M$ and $V$ are complex manifolds. Let $R$ be the branch locus.

We shall call $M$ a cyclic covering of $V$ if the following conditions are satisfied:

(i) For each $x \in R, \pi^{-1}(x)$ consists of one point.

(ii) The group of covering transformations of $M-\pi^{-1}(R)$ over $V-R$ is cyclic of order $k$.

(iii) If $k \neq 2$ then $R$ is connected.

We note that as a consequence of [Wk, Appendix] we have that $R$ will be nonsingular.

We now state

COROLlaRY 4.5. Suppose $X$ is an almost completely decomposable nonsingular algebraic surface and $C$ is an irreducible nonsingular hypersurface section of $X$ in some embedding $X \hookrightarrow C P^{N}$. Suppose $M$ is a $k$-fold cyclic branched covering of $X$ with branch locus $R \in|k C|$.

Then $M$ is almost completely decomposable.

Proof. By Theorem 1.2 of [Wk] there exists a line bundle $E$ on $X$ and a covering $\left\{V_{i}\right\}$ of $X$ over which $E$ is trivialized such that $M$ is diffeomorphic to the submanifold of $E$ defined by the equation $\xi_{i}^{k}=\phi_{i}$ where $\xi_{i}$ is the fiber coordinate of $E$ over $V_{i}$ and $\phi_{i}=0$ is the equation of $R$ in $V_{i}$. Furthermore using the corollary to Theorem 1.2 we see that $E$ is precisely the bundle [C] over $X$. Let $W$ be the projectivization of $E$. Then it is clear that $V \in|k X|$ on $W$ so that by our theorem it is A.C.D.

We recall that a 'double plane' is a 2-sheeted analytic branched cover of $C P^{2}$. Corollary 4.5 now gives us

COROllary 4.6. Suppose $V$ is an irreducible nonsingular double plane. Then $V$ is almost completely decomposable.

Proof. Since $V$ is nonsingular it is in fact a 2 -fold cyclic cover. But then by Corollary 4.5 it is A.C.D.

REMARK. In [MM1] we proved that the nonsingular hypersurfaces in $C P^{3}$ were all A.C.D. The hypersurface of degree $n, V_{n}$, thus gives us a sequence of 
A.C.D. algebraic surfaces whose 'vital statistics' (i.e., geometric genus $p_{g}(V)$ and signature $\sigma(V))$ vary with $n^{3}$. (Specifically $p_{g}\left(V_{n}\right)=1 / 6(n-1)(n-2)(n$ -3) and $\sigma(V)=-1 / 3 n\left(n^{2}-4\right)$.) The sequence of double-planes with ramification locus a curve $R$ of degree $2 r$ in $C P^{3}$ give rise to a sequence of A.C.D. algebraic surfaces $M_{r}$ with vital statistics varying with $r^{2}$. (In particular, $p_{g}\left(M_{r}\right)=1 / 2(r-1)(r-2)$ and $\sigma\left(M_{r}\right)=2\left(1-r^{2}\right)$.)

We also note that Corollary 4.5 provides a new (and different) proof that all hypersurfaces of degree $n$ in $C P^{3}$ are $A \subset D$. We extract this as a separate corollary.

COROLIARY 4.7 (COMPARE [MM1, 5]). Suppose $V_{n}$ is a nonsingular hypersurface of $C P^{3}$ of degree $n$.

Then $V$ is almost completely decomposable.

Proof. Since all nonsingular hypersurfaces of fixed degree are diffeomorphic we may assume $V_{n}$ is given by the equation $Z_{0}^{n}+Z_{1}^{n}+Z_{2}^{n}+$ $Z_{3}^{n}=0$ in $C P^{3}$. Projecting this surface onto the hyperplane $Z_{3}=0$ from the point $(0,0,0,1)$ makes $V_{r}$ into a cyclic $n$-sheeted cover of $C P^{2}$ with branch locus a nonsingular curve of degree $n$. Thus by Corollary $4.5 V_{n}$ is A.C.D.

Returning to Theorem 4.2 let us examine the divisors $m X+l S$ more closely. If $k$ is positive we note the following facts.

(1) If $D_{(m, l)}=m X+l S$ then if $m>0$ and $l>0$ then $D_{(m, l)}$ is a very ample divisor.

This follows almost immediately from the local form

$$
d_{\alpha} \zeta_{1 \alpha}^{n}-e_{\alpha} \zeta_{0 \alpha}^{m}=0
$$

for a nonsingular $V_{(m, l)} \in\left|D_{(m, l)}\right|$. This local representation shows us that $\left|D_{(m, l)}\right|$ separates points and it is easy to verify that it also separates infinitely near points and so as in $[\mathbf{H t}]\left|D_{(m, l)}\right|$ is very ample.

(2) The map $\Phi$ associated to the linear system $|X|$ maps $W$ onto a projective cone $\overline{C X} \hookrightarrow C P^{N+1}$ over $X \hookrightarrow C P^{N}$ with $\Phi: W \hookrightarrow \overline{C X}$ blowing down the 'section at $\infty$ ' of $W$ to the vertex of $\overline{C X}$.

This can also be seen by an explicit computation as above using the fact that if $V \in|X|$ and $X_{\infty}$ is the ' $\infty$-section' of $W$ then $Y \cap X_{\infty}=\varnothing$.

These observations then motivate the following:

THEOREM 4.8. Suppose $W$ is a compact analytic variety of complex dimension 3 and $V$ is an almost completely decomposable complex submanifold of $W$. Suppose $|V|$ is base point free and induces a holomorphic map $\Phi: W \rightarrow C P^{N}$ of $W$ onto some algebraic 3-fold $W^{\prime}$ such that $\Phi$ embeds $V$ onto a nonsingular hypersurface section $V^{\prime}$ of $W^{\prime}$ and is a diffeomorphism on some tubular neighborhood $T(V)$ of $V$ onto $T^{\prime}\left(V^{\prime}\right)$.

Then for any $n \geqslant 1$ 
(1) there exists a nonsingular element $V_{n} \in|n V|$,

(2) any nonsingular element of $|n V|$ is almost completely decomposable.

Proof. Since $V^{\prime}$ is a hypersurface section of $W^{\prime}$ it is clear that we can always find a nonsingular $V_{n}^{\prime} \in\left|n V^{\prime}\right|$ with $V_{n}^{\prime} \subset T^{\prime}\left(V^{\prime}\right)$. But then setting $\Phi^{-1}\left(V_{n}^{\prime}\right)=V_{n}$ we get the desired nonsingular element of $|n V|$. Furthermore since $V^{\prime}$ is a hypersurface section of $W^{\prime}$ it is clear that for any $n>1$ we can choose nonsingular $V_{n}^{\prime} \in\left|n V^{\prime}\right|, \quad V_{n+1}^{\prime} \in\left|(n+1) V^{\prime}\right|, \quad X_{n+1}^{\prime} \in\left|V^{\prime}\right|$, with normal crossing in $T^{\prime}\left(V^{\prime}\right)$. Clearly

$$
V_{n}^{\prime} \cdot V_{n+1}^{\prime} \cdot X_{n+1}^{\prime}=n(n+1)\left(V^{\prime}\right)^{3} \geqslant n(n+1)
$$

and either genus $\left(V_{n}^{\prime} \cap X_{n+1}^{\prime}\right)>0$ or $X_{n+1}^{\prime}$ is rational. Then the same argument used to prove Lemma 4.1 works here also and we can conclude that $V_{n}^{\prime}$ is A.C.D. for all $n$. But then clearly so is $V_{n}$ and since nonsingular linearly equivalent divisors of $W$ are diffeomorphic any nonsingular element of $|n V|$ is also A.C.D.

An immediate corollary is, of course,

COROLlary 4.9. Suppose $W$ is a compact analytic variety of complex dimension 3 and $V$ is an almost completely decomposable complex submanifold of $W$ which defines a very ample line bundle $[V]$ on $W$. Then $W$ is projective algebraic and for any $n \geqslant 1$

(1) there exists a nonsingular element of $V_{n} \in|n V|$,

(2) any nonsingular element of $V_{n} \in|n V|$ is almost completely decomposable.

Proof. Since very ample line bundles induce embeddings and $V$ is nonsingular it is easy to see that all the conditions of our theorem are satisfied.

\section{REFERENCES}

[GR] R. C. Gunning and H. Rossi, Analytic functions of several complex variables, PrenticeHall, Englewood Cliffs, N. J., 1965.

[Ht] Robin Hartshorne, Ample subvarieties of algebraic varieties, Lecture Notes in Math., vol. 156, Springer-Verlag, Berlin, 1970.

[H] J. F. P. Hudson, Piecewise linear topology, Benjamin, New York, 1969.

[KM] M. A. Kervaire and J. Milnor, Groups of homotopy spheres. I. Ann. of Math. (2) 77 (1963), 504-537.

[MM1] R. Mandelbaum and B. Moishezon, On the topological structure of non-singular algebraic surfaces in $C P^{3}$, Topology 15 (1976), 23-40.

[MM2] _ On the topology of algebraic surfaces, Trans. Amer. Math. Soc. (to appear).

[Mz], B. Mazur, Differential topology from the point of view of simple homotopy theory, Inst. Hautes Etudes Sci. Publ. Math. No. 15.

[Mi] J. Milnor, On simply connected 4-manifolds, Sympos. Internat. Topologica Algebraica, Mexico, 1958, pp. 122-128. 1974.

[RS] C. P. Rourke and B. J. Sanderson, Piecewise linear topology, Springer-Verlag, Berlin,

[Sh] I. Shafarevitch, Basic algebraic geometry, Springer-Verlag, Berlin, 1975. 
[S] N. E. Steenrod, Topology of fiber bundles, Princeton Univ. Press, Princeton, N. J., 1951.

[W1] C. T. C. Wall, Diffeomorphisms of 4-manifolds, J. London Math. Soc. 39 (1964), 131-140.

[W2] __, On simply connected 4-manifolds, J. London Math. Soc. 39 (1964), 141-149.

[Wk] J. J. Wavrik, Deformations of Banach coverings of complex manifolds, Amer. J. Math. 90 (1968), 926-960.

Department of Mathematics, Wetzmann Institute of SCIENCE, Rehovot, IsRabl

Current address: Department of Mathematics, University of Rochester, Rochester, New York 14618 